\title{
Medical Mycology dissertation topics require prioritisation among Postgraduate Microbiology trainees of Makerere University, Uganda.
}

Beatrice Achan ( $\sim$ beatriceachan@yahoo.co.uk)

Department of Medical Microbiology, College of Health Sciences, Makerere University, Kampala, Uganda https://orcid.org/0000-0001-9741-5429

\section{Gerald Mboowa}

Infectious Diseases Institute, College of Health Sciences, Makerere University, Kampala, Uganda

\section{Richard Kwizera}

Infectious Diseases Institute, College of Health Sciences, Makerere University, Kampala, Uganda

\section{David P. Kateete}

Department of Immunology and Molecular Biology, College of Health Sciences, Makerere University,

Kampala, Uganda

\section{Henry Kajumbula}

Department of Medical Microbiology, College of Health Sciences, Makerere University, Kampala, Uganda

Felix Bongomin ( $\nabla$ drbongomin@gmail.com )

Department of Medical Microbiology and Immunology, Faculty of Medicine, Gulu University, Gulu, Uganda

\section{Research Article}

Keywords: Medical Mycology, Dissertation, Postgraduate studies

Posted Date: March 9th, 2021

DOl: https://doi.org/10.21203/rs.3.rs-311293/v1

License: (c) (i) This work is licensed under a Creative Commons Attribution 4.0 International License.

Read Full License 


\section{Abstract}

Background: The unfortunate ongoing high burden of fungal diseases requires expertise in medical mycology.

Objective: The popularity of medical mycology dissertation topics among postgraduate microbiology trainees at the College of Health Sciences, Makerere University, Uganda was determined.

Methods. Dissertations submitted to the Departments of Medical Microbiology and Immunology \& Molecular Biology from 2011 through 2018 were reviewed retrospectively and the proportion of topics in medical mycology expressed as fractions of the total number of dissertations were analysed using descriptive statistics.

Results. A total of 152 dissertations were retrieved. Of these, only 5 (3.3\%) dissertations were on medical mycology compared to bacteriology $(50.7 \%, n=77)$, virology $(27.6 \%, n=42)$, parasitology $(14.5 \%, n=22)$ and immunology $(4.0 \%, n=6)$. Of the 5 dissertations on mycology, the fungal diseases studied by the postgraduate microbiology trainees were cryptococcal meningitis $(40 \%, n=2)$, Candidiasis $(20 \%, n=1)$, superficial mycoses $(20 \%, n=1)$ and invasive fungal diseases $(20 \%, n=1)$. The most common method that was used for studying the fungal diseases was the conventional culture methods $60 \%, n=3$.

Conclusion. Medical Mycology is not a popular research topic among postgraduate trainees of microbiology at Makerere University.

\section{Background}

Fungal diseases have emerged as important causes of morbidity and mortality worldwide ${ }^{1-3}$. The rising incidence of fungal diseases is due to increases in the number of susceptible individuals including people living with human immunodeficiency virus HIV/AIDS (PLWH), cancer patients, immunosuppressive therapy, organ transplant recipients and patients requiring critical care with prolonged hospitalisation 4,5 .

More than $90 \%$ of fungal-related deaths result from species that belong to one of the four genera, Cryptococcus, Candida, Aspergillus and Pneumocystis ${ }^{1-3}$. The species of these fungal genera cause a spectrum of diseases which range from superficial conditions of the outer keratinised layer of the skin to invasive fungal disease (IFD) of the bloodstream and/or deep seated organs such as the brain, heart, liver, lungs, spleen and the kidneys ${ }^{6}$. Of particular concern is the high mortality rate associated with invasive fungal infections which often exceeds $50 \%$ despite antifungal therapy 2,7 .

In Uganda, HIV/AIDS has underscored cryptococcal meningitis as a leading IFD ${ }^{8}$ where the 6-month survival remains $\leq 40 \%$ in routine antiretroviral and antifungal therapy ${ }^{9}$. One way to contribute to the fight against the burden of fungal diseases is to build capacity through training and research in medical mycology ${ }^{10}$. At Makerere University; the second best medical school in Africa, postgraduate students can choose to conduct research for their dissertations in medical mycology. Therefore, in this study, we aimed 
to determine the popularity of medical mycology topics among the microbiology postgraduate trainees at Makerere University.

\section{Materials And Methods Study Design and Setting}

This descriptive retrospective study was conducted between March and October of 2020, inclusive. Topics of dissertations of the Microbiology postgraduate trainees of Master of Medicine in Microbiology (M. Med) and Master of Immunology and Clinical Microbiology (MICM) of Makerere University from 2011 through 2018 were reviewed. The study sites were the Departments of Medical Microbiology and Immunology and Molecular Biology, School of Biomedical Sciences, College of Health Sciences, Makerere University.

\section{Data management and analysis}

Data on the dissertations including year, research topic, organism/category of organism and study methods used were entered in Microsoft Office Excel 2016 (Microsoft Corp., Redmond, WA, USA). The research methods used in the theses were further classified as microscopy, culture, molecular biology, antigen detection and serology. The data were analysed using ggplot2 package in $\mathrm{R}$ statistical computing software and analysed for the number of theses, frequency of medical microbiology sub-disciplines of Bacteriology, Virology, Parasitology, Mycology and Immunology.

\section{Ethical issues}

The authors confirm that the ethical policies of the journal, as noted on the journal's author guidelines page, have been adhered to. No human participants were involved in the study. Secondary data were largely available from Makerere University archive and so no ethical clearance was needed. The student names were anonymised, and only study numbers were used for identification.

\section{Results}

A total of 152 dissertations that were submitted to the Departments of Medical Microbiology and, Immunology and Molecular Biology from 2011 to 2018 were reviewed (Fig. 1). The least number of theses was recorded in 2014 at 5.9\% (9/152) while 2018 saw the highest number of submitted theses at $21.1 \%$ (32/152) (Fig. 1) over the eight years. The most popular theses sub-discipline was bacteriology at $50.7 \%(77 / 152)$ while mycology was the least researched at only 3.3\% (5/152) (Fig. 2). The rest of the sub-disciplines included: virology $27.6 \%(42 / 152)$, parasitology at $14.5 \%(22 / 152)$, and immunology of $4.0 \%(6 / 152)$ (Fig. 2).

The most common methods used in the dissertations were immunology $49.3 \%$ (75/152), culture $23 \%$ (35/152) and molecular biology $22.3 \%$ (34/152) while bioinformatics and microscopy were the least used $4 \%(6 / 152)$ and $1.3 \%(2 / 152)$, respectively (Fig. 3.). 
Of the 5 mycology dissertations, 2 of them were on cryptococcal meningitis diseases $(40 \%, n=2)$, while for the 3 remaining dissertations were each written on superficial mycoses $(20 \%, n=1)$, candidiasis $(20 \%$, $\mathrm{n}=1)$ and invasive fungal diseases $(20 \%, \mathrm{n}=1)$ (Table 1$)$.

All the methods used for mycology dissertations were phenotypic based; culture $(n=3)$ and immunology $(n=2)($ Table 1).

Table 1

Fungal diseases studied by postgraduate trainees who wrote mycology dissertations over the years from 2012 to 2019 and the methods used.

\begin{tabular}{|lll|}
\hline Year & Fungal disease & Methods \\
\hline 2012 & Superficial mycoses & Culture \\
\hline 2012 & Cryptococcal meningitis & Immunology \\
\hline 2014 & Cryptococcal meningitis & Immunology \\
\hline 2014 & Candidiasis & Culture \\
\hline 2019 & Invasive fungal diseases & Culture \\
\hline
\end{tabular}

\section{Discussion}

Fungal infections continue to impose a significant threat, globally ${ }^{1-3}$ exacerbated by a plethora of challenges faced in the field of medical mycology (10). The present study to determine the popularity of medical mycology research topics among postgraduate microbiology trainees in one of Africa's leading University in Uganda, showed that only 5 (3.3\%) dissertations were written on fungal diseases. This means that medical mycology was the least researched dissertation topic at Makerere University.

One possible explanation to the limited research on fungal diseases among postgraduate trainees is the lack of formal training in medical mycology. I am the first and only formally trained mycologist at Makerere University. A result of the previous deficit is that the teaching of mycology is only a minor section; three credit units (maximum being five) in the training of postgraduate M. Med. students at Makerere University. Moreover, in the MICM program, medical mycology is not taught as a separate course unit but an integral component of fundamentals of microbiology course unit. With the limited 
mycology content in the medical microbiology postgraduate programs, perhaps, the student's research dissertations were not guided by their interests but faculty research projects in which their studies were nested. This inference is supported by the fact that the most researched fungal diseases were cryptococcal meningitis $(40 \%, \mathrm{n}=2)$ which is the leading invasive fungal disease highlighted by HIV/AIDS in Uganda ${ }^{11}$.

Makerere University, College of Health Sciences through the Infectious Diseases Institute is an internationally recognised research hub for cryptococcal meningitis. However, as elsewhere in other resource limited countries, capacity for research in other fungal diseases is just improving in Uganda ${ }^{10}$.

Additionally, we analysed the frequency of methods used for research in the theses (Fig. 3). Most methods that were used were molecular biology techniques. However, molecular techniques are not yet well developed for diagnosis of fungal diseases. Significantly, in low- and middle -income countries, like Uganda, where molecular tests are prohibitively costly, the predominant methods for diagnosis of fungal diseases are microscopy and culture based phenotypic techniques. In all the medical mycology theses, only phenotypic methods were used. Therefore, it is possible that the limited laboratory methods could have affected the choice of the students to conduct research in fungal diseases.

Another obvious and true possibility is that students lacked funding to explore their research interests in fungal diseases. Unlike other fields, dedicated funding for the training of students in medical mycology from major funding bodies is only emerging, where at present, the EU EDCTP is funding an early career fellowship at Makerere University.

Our findings are consistent with a report from a recent inaugural European Molecular Biology Organisation meeting on AIDS-related mycoses that listed training needs, requirements for laboratory testing methods and increased funding as priority areas for improved mycology research in low- and middle- income settings ${ }^{10}$. A study from Gulu University in Uganda, also showed that using freely available online materials on medical mycology for teaching and learning can enhance capacity building in medical mycology ${ }^{12}$. Therefore, there is need to establish a stand-alone formal medical mycology postgraduate programs in Uganda. Following, the Department of Medical Microbiology in collaboration with Manchester University, UK has developed a curriculum for MSc Medical Mycology to be hosted at Makerere University. It is expected that the program will lead to a steep rise in the number of medical mycologists in Uganda. The collaboration between Makerere University and Manchester University are also working to ensure that teaching materials can be made available online. The findings of our study are in agreement with the report by Brown et $a^{10}$ on improving mycology expertise in developing countries through training.

\section{Conclusion}

We report a low interest in fungal disease research among postgraduate microbiology trainees of Makerere University, Uganda, possibly due to lack of a formal medical mycology training program. There 
is need to establish and fund a medical mycology program at Makerere University.

\section{Declarations}

\section{Authors' contributions.}

B.A designed the study, collected the data, coordinated the research, performed data analysis, wrote and reviewed the manuscript. GM analysed the data and reviewed the manuscript. RK reviewed the manuscript. DPK provided administrative support in archiving the data and reviewed the manuscript. HK provided leadership support and reviewed the manuscript. FB perceived the study, designed the study, coordinated the research, wrote and reviewed the manuscript. All authors read and approved the final manuscript.

\section{Acknowledgement.}

The study did not receive specific funding. We are grateful to Geraldine Nalwadda who retrieved the list of the dissertations submitted to the departments. BA is a NURTURE (NIH/International Forgarty Centre) Fellow under the NIH grant D43TW010132, has EDCTP fellowship (TMA2018CDF-2371) and is MAKRIF faculty grant holder. RK is a PhD fellow supported through the DELTAS Africa Initiative grant \# DEL-15011 to THRiVE-2, from Wellcome Trust grant \# 107742/Z/15/Z and the UK government. The funders had no roles in the study design, data collection, analysis, decision to publish or preparation of the manuscript.

\section{Conflict of interest.}

All authors have no conflict of interest.

\section{References}

1. Bongomin F, Gago S, Oladele R, Denning D. Global and Multi-National Prevalence of Fungal Diseases -Estimate Precision. J Fungi. 2017;3(4):57. doi:10.3390/jof3040057

2. Brown GD, Denning DW, Gow NAR, Levitz SM, Netea MG, White TC. Hidden killers: Human fungal infections. Sci Trans/ Med. 2012;4(165):1-9. doi:10.1126/scitranslmed.3004404

3. Netea MG, Brown GD. Fungal infections: The next challenge. Curr Opin Microbiol. 2012;15(4):403405. doi:10.1016/j.mib.2012.07.002

4. Richardson M, Lass-Flörl C. Changing epidemiology of systemic fungal infections. Clin Microbiol Infect. 2008;14(SUPPL. 4):5-24. doi:10.1111/j.1469-0691.2008.01978.x

5. Vallabhaneni S, Mody RK, Walker T, Chiller T. The Global Burden of Fungal Diseases. Infect Dis Clin North Am. 2016;30(1):1-11. doi:10.1016/j.idc.2015.10.004

6. Kullberg BJ, Arendrup MC. Invasive Candidiasis. N Engl J Med. 2015;373(15):1445-1456. doi:10.1056/NEJMra1315399 
7. Ellis J, Bangdiwala AS, Cresswell F V, et al. The Changing Epidemiology of HIV-Associated Adult Meningitis, Uganda 2015-2017. Open forum Infect Dis. 2019;6(10):ofz419. doi:10.1093/ofid/ofz419

8. Parkes-Ratanshi R, Achan B, Kwizera R, Kambugu A, Meya D, Denning DW. Cryptococcal disease and the burden of other fungal diseases in Uganda; Where are the knowledge gaps and how can we fill them? Mycoses. 2015;58:85-93. doi:10.1111/myc.12387

9. Longley N, Muzoora C, Taseera K, et al. Dose response effect of high-dose fluconazole for HIVassociated cryptococcal meningitis in southwestern Uganda. Clin Infect Dis. 2008;47(12):15561561. doi:10.1086/593194

10. Brown GD, Meintjes G, Kolls JK, et al. AIDS-related mycoses: the way forward. Trends Microbiol. 2014;22(3):107-109. doi:10.1016/j.tim.2013.12.008

11. Kwizera R, Bongomin F, Lukande R. Deep fungal infections diagnosed by histology in Uganda: a 70year retrospective study. Med Mycol. Published online 2020:1-9. doi:10.1093/mmy/myaa018

12. Bongomin F, Erima B, Kwizera R, Odongo-Aginya El. Online Learning Resources Enhanced Teaching and Learning of Medical Mycology among Medical Students in Gulu University, Uganda. Educ Res Int. 2020;2020:1-5. doi:10.1155/2020/9468241

\section{Figures}

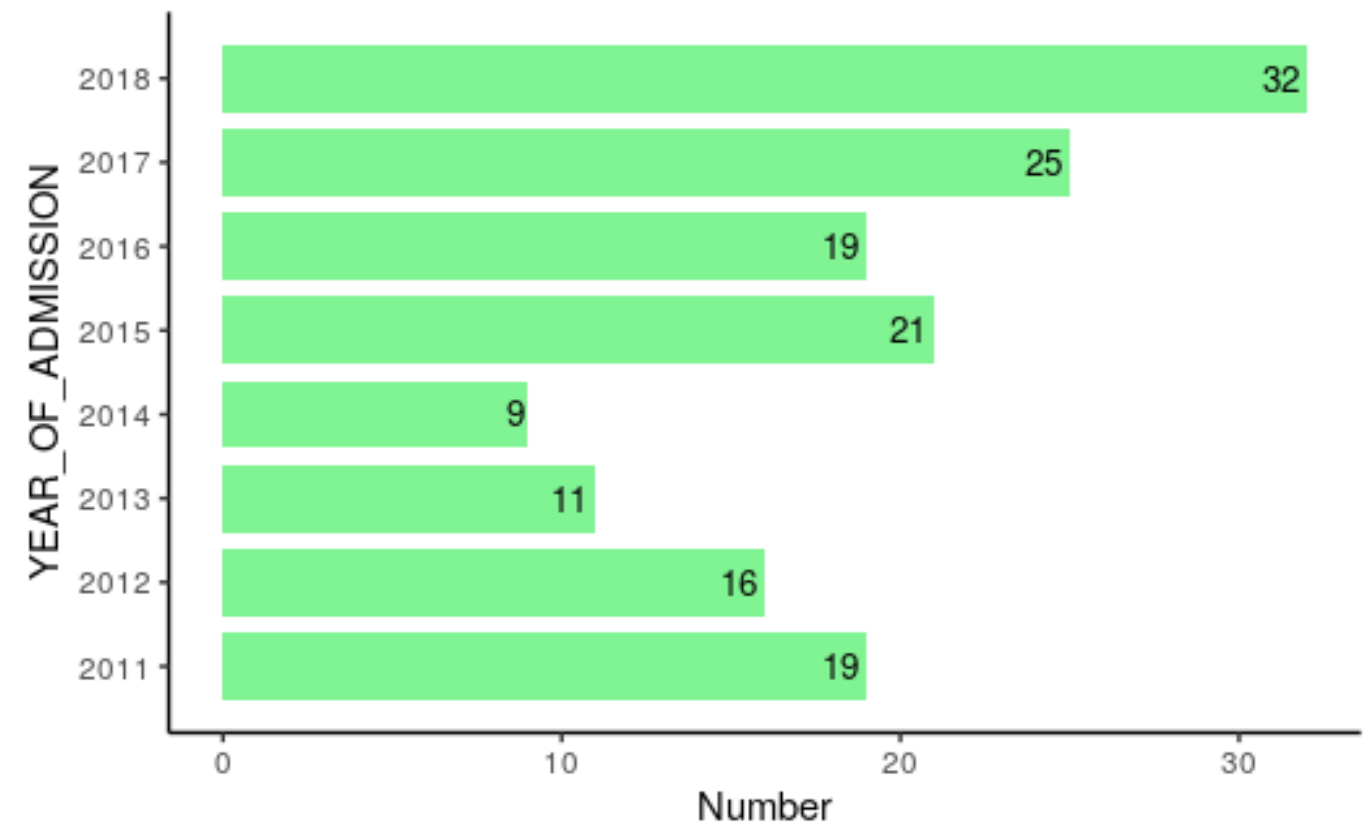

\section{Figure 1}

The number of theses submitted from 2011-2018 The years 2014 and 2018 had the lowest and highest numbers of theses, respectively. 


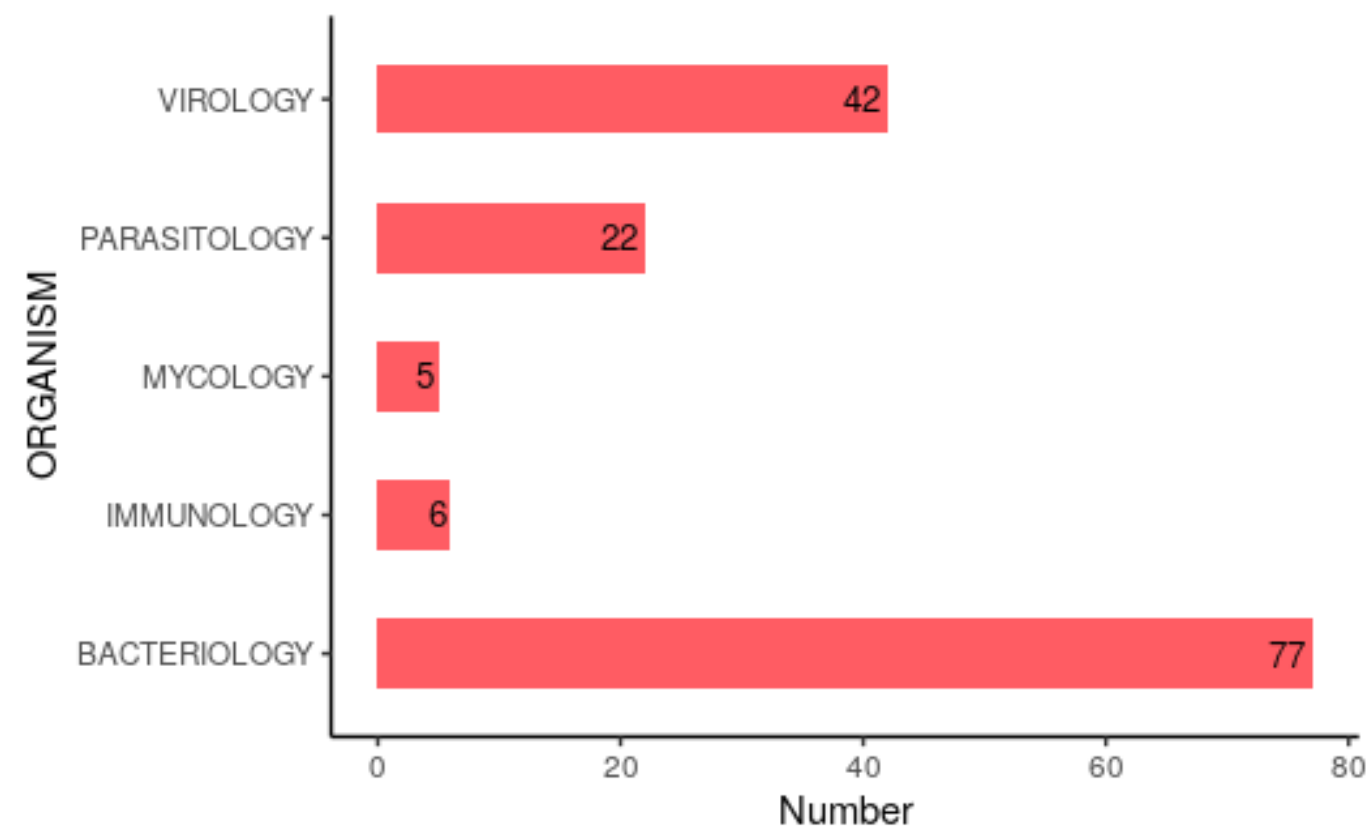

\section{Figure 2}

Frequency of sub-disciplines of the dissertations submitted. Bacteriology projects are more popular among postgraduate microbiology trainees

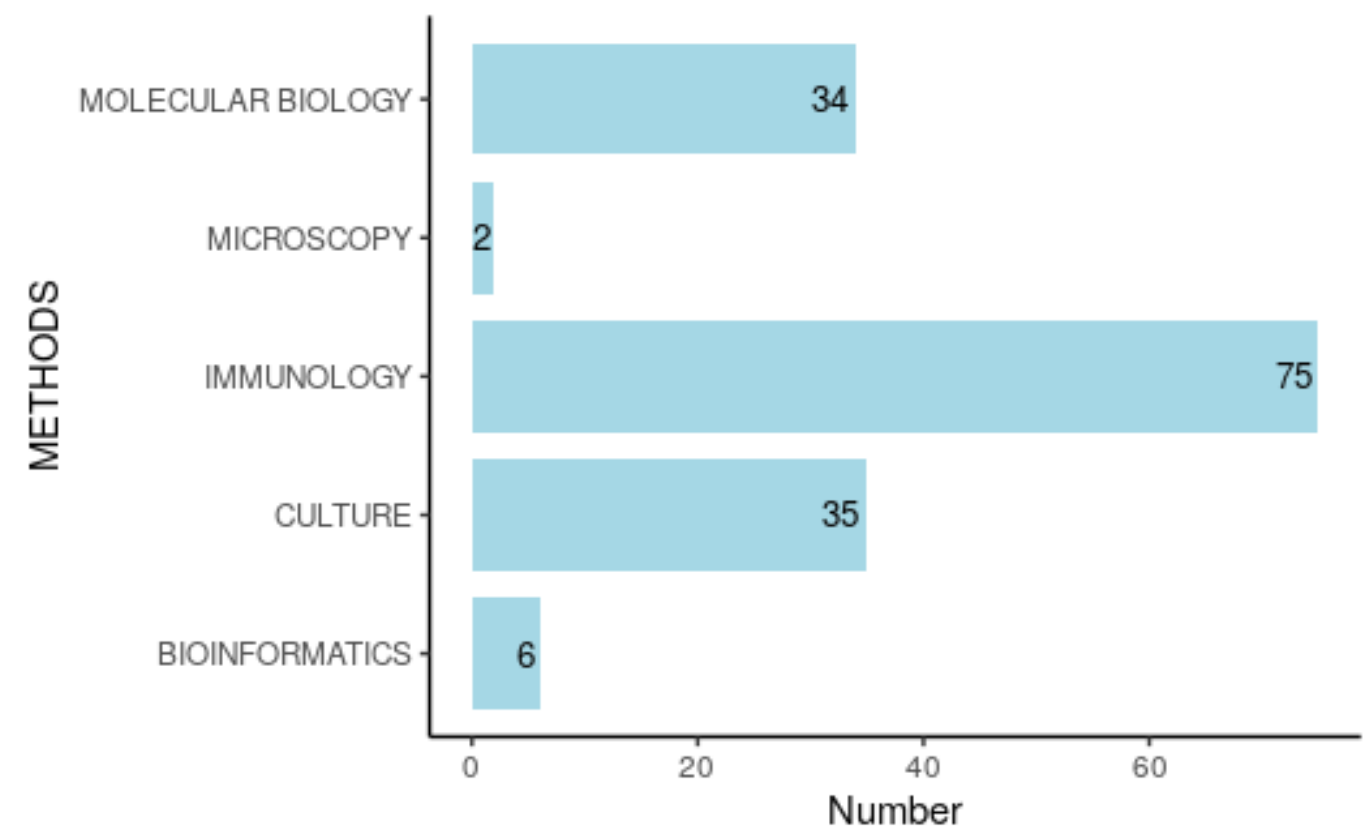

Figure 3

Methods used for data collection in the theses. Immunological techniques were commonly used.

\section{Supplementary Files}

This is a list of supplementary files associated with this preprint. Click to download. 
- SupplemenaryFile.xls

Page 9/9 